\title{
Querying Image Database by Video Content*
}

\author{
C. H. WANG, H. C. LIN ${ }^{* 1}$, C. C. SHIH", H. R. TYAN ${ }^{\dagger}$, C. F. LIN" \\ AND H. Y. MARK LIAO \\ Institute of Information Science \\ Academia Sinica \\ Taipei, 115 Taiwan \\ ${ }^{* 1}$ Department of Information Management \\ Chang Jung Christian University \\ Tainan Hsien, 711 Taiwan \\ ** Department of Ecology and Evolution \\ University of Chicago, USA \\ ${ }^{\dagger}$ Department of Information and Computer Engineering \\ Chung Yuan Christian University \\ Chungli, 320 Taiwan \\ ${ }^{\dagger}$ Department of Computer Science and Engineering \\ Yuan Ze University \\ Chungli, 320 Taiwan
}

\begin{abstract}
A content-based image retrieval (CBIR) system using the features extracted from a video clip is proposed in this paper. The proposed CBIR system is directly applied to access a fish database collected and managed by the Institute of Zoology, Academia Sinica, Taiwan. The major contribution of this work is three-fold: (1) the proposed query-by-video-content scheme can overcome the problems caused by non-rigid motions, serious deformations, and partial occlusions of target objects; (2) the proposed scheme can tolerate different imaging environments; (3) the proposed representation scheme is invariant to translation, scaling, and rotation. Experimental results have confirmed the effectiveness of the proposed CBIR system.
\end{abstract}

Keywords: content-based image retrieval (CBIR), shape similarity retrieval, query by video content, curvature scale space (CSS), CSS-based representation and matching

\section{INTRODUCTION}

Content-based image retrieval (CBIR) has become an active research area in recent years [1-9]. Since the number of digital images from a broad range of sources is growing rapidly, an efficient technique that can be applied to find target images within seconds is urgently required. Using content-based techniques, a user can present the content of interest as a query. The content may be color, texture, shape, or the spatial layout of a target image. Images that exhibit "perceptual similarity" with the query can be found in the repertory. Some readily available commercial systems for this purpose include QBIC (Query By Image Content), Virage, RetrievalWare, and so on. There are also some good

Received January 17, 2003; revised May 15, 2003; accepted June 16, 2003.

Communicated by Kuo-Chin Fan.

* This study was supported partially by the National Science Council, R.O.C. under Grant

NSC89-2213-E-001-021, NSC89-2218-E-001-008, and NSC90-2213-E-309-004. 
systems that have been developed by academic researchers, such as Photobook, WebSEEk, Netra, MARS (Multimedia Analysis and Retrieval System), and so on. Detailed surveys on CBIR systems can be found in [1-9].

Among the different types of image contents, the shape feature usually plays an important role due to its relatively unique property. Humans can easily identify an object via its rough shape. This property makes the shape feature very popular in numerous CBIR systems. Generally speaking, shape-based retrieval systems can be categorized into two classes, depending on the types of features used to describe shapes. They are either region-based or contour-based systems [10]. In region-based systems, two shapes are considered similar if they have similar spatial distributions of pixels. In contour-based systems, on the other hand, two shapes are considered similar if they have similar silhouettes. A number of works that used shape as the feature can be found in [11-22]. The Photobook system [11, 12] utilizes the finite element method to solve point correspondence and matching problems. The similarity between two distinct shapes is derived by computing the deformation energy. Another famous system that is worth mentioning is IBM's QBIC system [13-17]. The QBIC system adopts some global features, including area, circularity, eccentricity, major axis orientation, and moment invariants, to represent a shape boundary. The similarity between a query and each database image is calculated based on a weighted Euclidean distance in a multi-dimensional feature space. Since global features are very sensitive to the local variation of a given shape, a representation based on global features may result in unsatisfactory retrieval. To overcome this problem, Huang and Huang [18] proposed using the junction points and curvature extrema of a sketched object as feature points. In [19], Grosky and Lu proposed using 8-neighbor chain codes to represent a shape boundary. In this method, the similarity between two shapes can be defined by the length of a shift-window and the longest $q$-generalized common substrings of their corresponding strings.

In this paper, we propose a new CBIR system that can be directly applied to access a fish database collected and managed by the Institute of Zoology, Academia Sinica, Taiwan. The proposed CBIR system adopts completely different features as the basis for matching. The features are extracted from a sequence of images instead of a single image. In a conventional CBIR system, a user may query an image database using features extracted from a single image. The query-by-a-single-image scheme may suffer from a number of problems in the retrieval process. These problems include the following: (1) The imaging condition for a query image is not identical to that of database images. In this case, features extracted from the same target may be completely different. (2) Since a fish may swim in a non-rigid manner, and may sometimes turn at an extreme angle, it is difficult to characterize a fish using only one view. In order to tackle the above-mentioned problems, we decided to use features extracted from an image sequence to make the retrieval process more reliable. The proposed CBIR system is composed of two processes, including a model database construction process and a matching process. During the model database construction process, the foreground portion of every database image is first segmented from the background and then represented as a sequence of vertices ordered clockwise along its boundary. The boundary length is then normalized so that the scale problem can be overcome. In order to represent a fish, the fish boundary is smoothed gradually by Gaussian functions, and the corresponding curvature scale space (CSS) image is then created. The CSS image contains several arches 
representing the locations of curvature zero crossings (i.e., inflection points) on the smoothed fish boundary. Maxima points on the arches which form a feature vector are extracted, regularized, and stored in the database.

During the matching process, a user can choose an image sequence of a fish to query its detailed information. Each image in an image sequence is processed in the same way as we did to process a database image. However, because the pose of the fish may change from image to image, due to movement, fish images may be classified into three types, including left, right, and front (or rear) view images. We retain images having left and right views as those with valid poses during the matching process. The images that happen to be the front (or rear) views are considered useless and discarded. Finally, the c-means algorithm is executed, and the average maxima points are derived from a video clip. The average maxima points are used as a representative feature vector for making a comparison with those of each database image. Database images that are similar to the contents of the image sequence are then retrieved and displayed according to their matching scores. The proposed CBIR system is illustrated in detail in Fig. 1. The advantages of the proposed CBIR system are its invariance to translation, scaling, and rotation during the matching process. Moreover, the problems caused by non-rigid fish motion, serious deformations, and partial occlusions can be overcome.

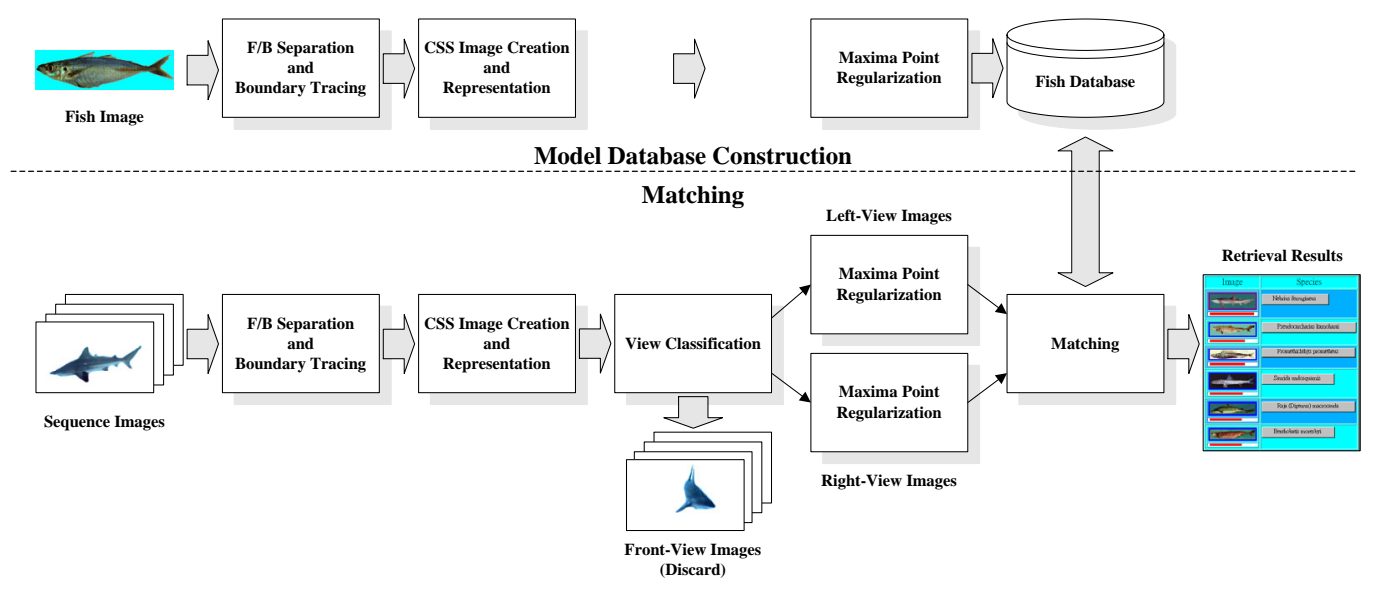

Fig. 1. The system overview.

The rest of this paper is organized as follows. Section 2 describes the model database construction process in detail. Section 3 details how the matching process is designed and executed. Section 4 presents the experimental results. Conclusions are drawn in section 5 .

\section{MODEL DATABASE CONSTRUCTION PROCESS}

This section will describe the processes needed to construct a model database. These processes include foreground/background separation, CSS-based representation, 
and regularization. They are, respectively, described in sections $2.1,2.2$, and 2.3.

\subsection{Foreground/Background Separation}

The foreground/background separation problem is ill-posed because it is by its nature a segmentation problem. In our early work on foreground/background separation [21], we assumed that a fish in an image is always captured by a mouse and located at the center part. Therefore, we can select 25-30 pixels from the upper-left portion of a bounding rectangle and assume that these pixels will form a Gaussian distribution. Using the mean and standard deviation of this distribution as the bases for deriving the thresholds, we may start a region growing process. If the difference between the gray level or color of a pixel and the mean value does not exceed two times of the standard deviation, we may consider this pixel as a background pixel; otherwise, it is a foreground pixel. We are able to separate correctly about $80 \%$ of fish images of this kind.

After the separation process, a simple boundary tracing method is used so that the fish can be represented as a sequence of points ordered clockwise along its boundary. To normalize the boundary length and eliminate the scale problem during the matching process, the fish boundary is re-sampled into 250 points with equal distances.

\subsection{CSS-Based Representation}

A good representation scheme is required to characterize the silhouette of a separated fish; it should be sufficiently compact while still preserving shape characteristics at various semantic levels [23]. In [24, 25], Witkin proposed a scale-space approach to determining spread parameters for smoothing a given signal. Gaussian convolution at gradually increasing spread parameters is first applied to the signal. Then, zero crossings in the second derivatives of the smoothed signal are plotted in the fingerprint (i.e., the CSS image), which characterizes the behavior of zero crossings in the signal as the spread parameter increases. Based on the above-mentioned scale-space approach, Abbasi et al. [22] proposed a CSS image representation scheme for shape similarity retrieval. A CSS image contains several arches representing the behavior of curvature zero crossings on a smoothed silhouette. The maxima points on these arches can be extracted and used as a feature vector to represent the shape. In what follows, we will introduce the CSS-based representation scheme that can be used to describe a closed silhouette.

Let $\left\langle(x(u), y(u)) \in \mathbf{Z}^{2} \mid 1 \leq u \leq n\right\rangle$ denote a shape boundary, where $u$ is the arc length (in points) and $n$ is the number of points on the boundary. The curvature function, $k(u)$, of the shape boundary is defined as [21]

$$
k(u)=\frac{\dot{x}(u) \ddot{y}(u)-\ddot{x}(u) \dot{y}(u)}{\left[\dot{x}^{2}(u)+\dot{y}^{2}(u)\right]^{3 / 2}},
$$

where $\dot{x}(u) \quad(\dot{y}(u))$ and $\ddot{x}(u) \quad(\ddot{y}(u))$ denote the first and second derivatives of $x(u)(y(u))$, respectively. To compute $\dot{x}(u)$ and $\ddot{x}(u)$, a simple method is applied that uses finite differences to approximate the differentiation as follows [26]: 


$$
\begin{aligned}
& \dot{x}(u)=x(u+1)-x(u-1), \\
& \ddot{x}(u)=x(u+1)-2 x(u)+x(u-1) .
\end{aligned}
$$

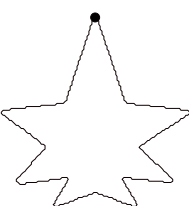

(a)

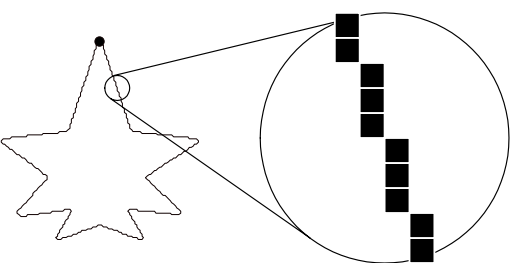

(b)

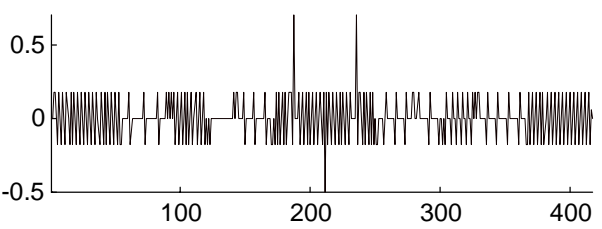

(c)

Fig. 2. Computing curvature using finite differences: (a) a shape boundary; (b) false convexity and concavity; (c) the curvature function.

$\dot{y}(u)$ and $\ddot{y}(u)$ can be obtained in the same way. Fig. 2 shows how the curvature function is computed using finite differences. Fig. 2(a) is a shape boundary with 417 points, where "•" indicates the starting point from which the curvature is computed clockwise. Although the shape boundary looks smooth, there exist false local convexities and concavities along the digitized curve, as shown in Fig. 2(b); the computed curvature values are very ragged, as shown in Fig. 2(c). As a result, detecting zero crossings based on a curve described by the curvature function becomes a very difficult task.

To overcome the above problem, we first convolve $x(u)$ and $y(u)$ with a 1D Gaussian function and then compute the curvature function. Let $X(u, \sigma)$ be the convolution of $x(u)$ with a $1 \mathrm{D}$ Gaussian function $g(u, \sigma)$, where

$$
g(u, \sigma)=\frac{1}{\sqrt{2 \pi} \sigma} \exp \left[\frac{-(u-m)^{2}}{2 \sigma^{2}}\right]
$$

Here, $m$ and $\sigma$ denote the mean and the spread parameter, respectively. Let $X(u, \sigma)$ denote the convolution of $x(u)$ with $g(u, \sigma)$. Then we have

$$
\begin{aligned}
X(u, \sigma) & =x(u) * g(u, \sigma) \\
& =\int_{-\infty}^{\infty} x(u) g(u-\mu, \sigma) d \mu
\end{aligned}
$$




$$
=\int_{-\infty}^{\infty} x(u) \frac{1}{\sqrt{2 \pi} \sigma} \exp \left[\frac{-(u-\mu)^{2}}{2 \sigma^{2}}\right] d \mu
$$

where "**" denotes the convolution operation with respect to $u$, and $\mu$ is a dummy variable. Let $\dot{X}(u, \sigma)$ and $\ddot{X}(u, \sigma)$ denote, respectively, the first and second partial derivatives of $X(u, \sigma)$ with respect to $u$. Since the Gaussian function is differentiable, $\dot{X}(u, \sigma)$ and $\ddot{X}(u, \sigma)$ can be derived as follows [21]:

$$
\begin{aligned}
\dot{X}(u, \sigma) & =\frac{\partial X(u, \sigma)}{\partial u} \\
& =\frac{\partial[x(u) * g(u, \sigma)]}{\partial u} \\
& =x(u) * \frac{\partial g(u, \sigma)}{\partial u} \\
\ddot{X}(u, \sigma) & =x(u) * \frac{\partial^{2} g(u, \sigma)}{\partial u^{2}},
\end{aligned}
$$

The partial derivatives $\dot{Y}(u, \sigma)$ and $\ddot{Y}(u, \sigma)$ can be derived in the same way. Therefore, the smoothed curvature function, denoted as $K(u, \sigma)$, at the value of $\sigma$ (the spread parameter) can be computed as follows [21]:

$$
K(u, \sigma)=\frac{\dot{X} \ddot{Y}-\ddot{X} \dot{Y}}{\left[\dot{X}^{2}+\dot{Y}^{2}\right]^{3 / 2}} .
$$

The positive and negative values of $K(u, \sigma)$ indicate the convexity and concavity, respectively. If the value of $K$ is equal to zero, its corresponding boundary point is called a zero crossing. The number and locations of zero crossings on the shape boundary depends on the value of $\sigma$. As $\sigma$ increases, the shape boundary shrinks and becomes smoother, and the number of zero crossings decreases. When $\sigma$ is sufficiently large, the shape boundary becomes a convex hull, and zero crossings no more exist.

If we plot the locations of zero crossings in the $u-K-\sigma$ space as $\sigma$ increases, a 3D CSS illustration can be generated. Figs. 3(a) and 3(b) show a fish and its corresponding 3D CSS illustration, respectively. In our experiments, $\sigma$ was initialized as 1 , and at each level, $\sigma$ was increased by 0.1 . The projection of a 3D CSS illustration on the $u-\sigma$ plane is known as a CSS image, as shown in Fig. 3(c). The arches in a CSS image reflect the changes of convexities, concavities, and zero crossings on the smoothed shape boundary. The intersections of a horizontal line (i.e., $y=\sigma$ ) and the arches in a CSS image indicate the locations of zero crossings on a smoothed shape boundary. The zero crossings are displayed in red. Between two neighboring zero crossings, the green and blue intervals indicate the corresponding convex and concave curves, respectively. As a shape boundary gets smoother and smoother, the neighboring zero crossings may get closer to each 


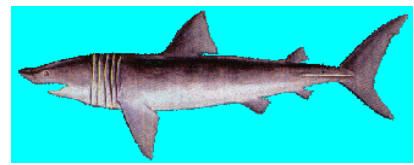

(a)

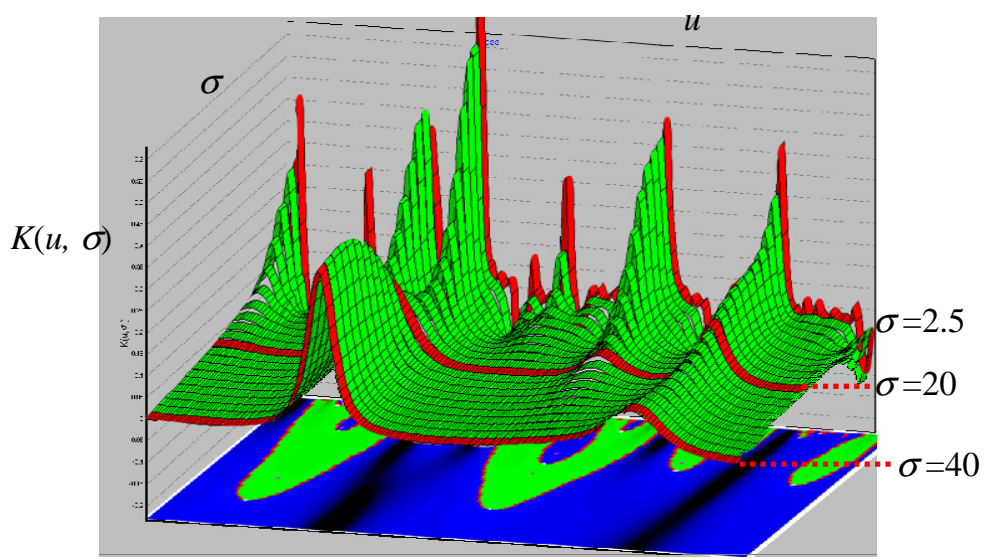

(b)

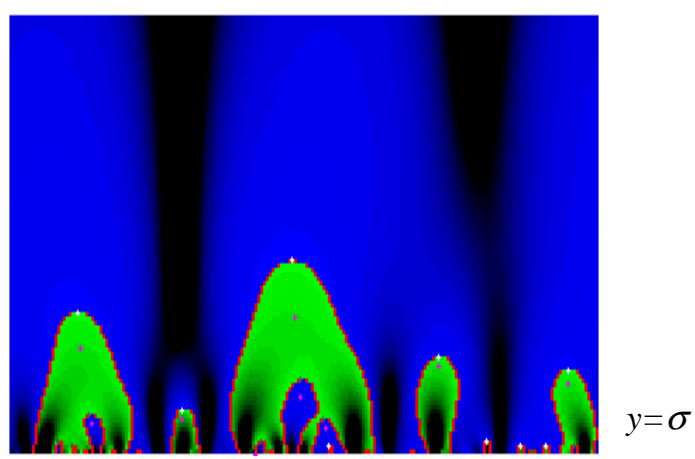

(c)

Fig. 3. Creation of a CSS image: (a) a fish; (b) the corresponding 3D CSS illustration; (c) the corresponding 2D CSS image.

other and form a complete arch in the CSS image. The location where the two zero crossings meets indicates the maximum point of this arch. On the other hand, the height of an arch reflects the depth and width of the corresponding convex (or concave) curve on the shape boundary. The deeper or wider the convex (or concave curve) is, the larger the magnitude of the maximum point.

\subsection{Regularization of a Representation}

Recall that the boundary length of a fish is always adjusted to 250 points. Therefore, our fish representation scheme is invariant to translation and scaling. However, there are 
two potential problems that may severely affect the performance of our approach. First, the representation may be totally different if the starting point is different. Second, a shallow concavity may generate a false maximum point, thus influence the accuracy.

To tackle the above problems, some steps have to be taken. In this paper, we propose a new method to uniquely determine a starting point. The direction toward where the fish moves can also be detected. As the value of $\sigma$ increases, the fish boundary changes from fine resolution to coarse resolution gradually. When $\sigma$ is sufficiently large, the shape boundary becomes a convex hull, and all zero crossings vanish. Fig. 4 shows three fish boundaries which correspond to $\sigma=2.5,20$, and 40, respectively. It is obvious that the fish boundary degenerates into a "quasi-ellipse" at $\sigma=40$, as shown in Fig. 4(c). The curve corresponds to $K(u, 40)$, a non-negative function, and contains only two local maxima. The boundary point located at the first local maximum corresponds to the fish tail, whereas the boundary point located at the second local maximum corresponds to the fish head. Between the two boundary points, the upper curve corresponds to the fish back, and the lower curve corresponds to the fish belly. Although the fish may swing its tail fin to move forward, we have found that the relative position of the fish head and tail is kept insensitive to updates in the CSS image, as shown in Fig. 5. The white and gray arrows indicate the positions of fish head and tail, respectively. Assume that $\sigma_{0}$ is the smallest $\sigma$ at which all zero crossings vanish from the CSS image. We use $\sigma_{i}=1.25 \sigma_{0}$ to smooth the curvature function and obtain a degenerated boundary. The boundary point with the second curvature maximum (i.e., the fish head) can be regarded as the starting point. This point also indicates the direction toward which the fish moves. Figs. 6(a) and 6(b) show the CSS image of a fish and its curvature function at $\sigma_{i}$, i.e., $K\left(u, \sigma_{i}\right)$. The curvature function contains two local maxima, corresponding to the fish tail and head, respectively. Fig. 6(c) shows the fish boundary at $\sigma_{i}$. When the starting point of a fish boundary is determined, its representation can be shifted in a circular manner as follows: Assume that the $u$-th boundary point, where $1<u \leq 250$, corresponds to the second local maximum (i.e., the fish head). The maxima points between $u$ and 250 are shifted to the left and fall into the interval between 1 and $251-u$, whereas those located between 1 and $u-1$ are shifted to the right and fall into the interval between $252-u$ and 250 . After the circular shift, the new representation can overcome the rotation problem, as shown in Fig. 7.

A shallow concavity is a very wide, but not deep, concave (or convex) curve on a shape boundary. A deep shallow concavity may create a very high arch in a CSS image. It is well known that this situation will create a false maximum point. Therefore, if a raw representation is used during the matching process, a shallow concavity may be matched with a deep one. The problem of shallow concavities can be overcome by adjusting the heights of maxima points within arches [27].

\section{MATCHING PROCESS}

In this section, we shall explain in detail how the matching process is executed. In section 3.1, we shall present how our CSS-based model can be used to describe a moving fish. Then, in section 3.2, we shall describe how the proposed CSS-based model can be used to perform matching. 

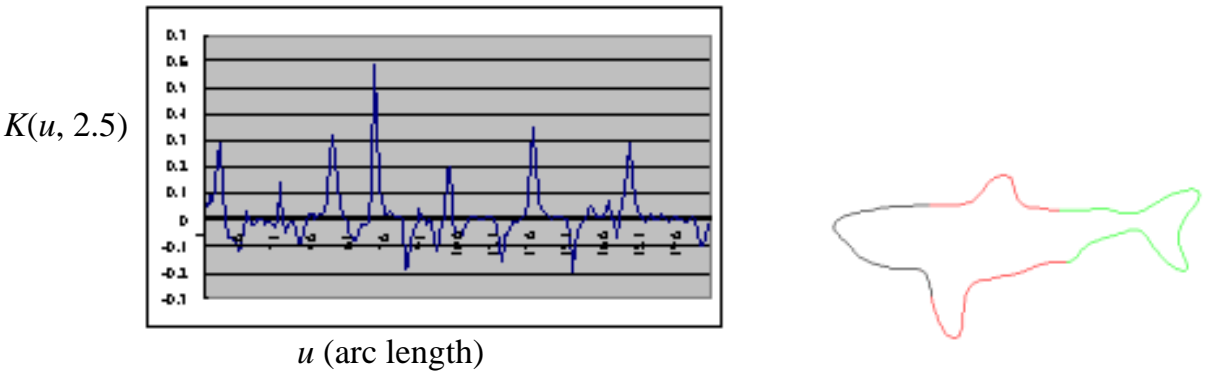

(a)

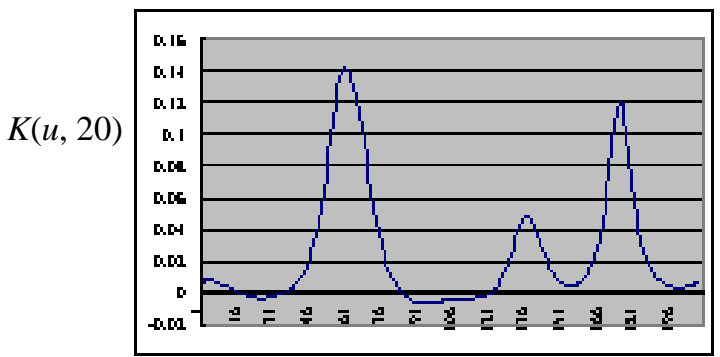

$u$ (arc length)

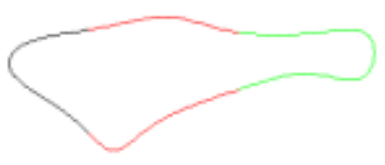

(b)

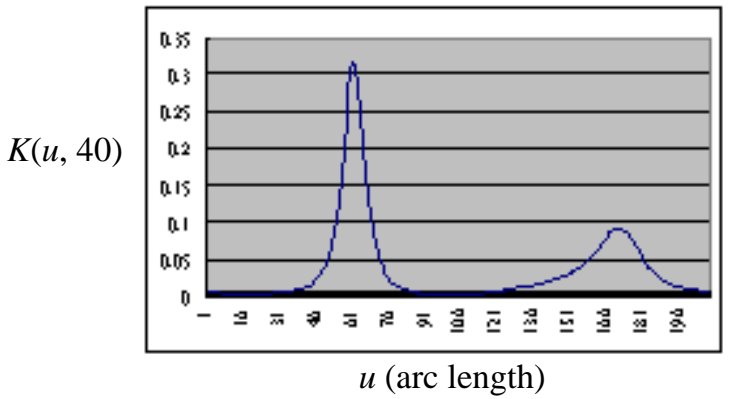

(c)

Fig. 4. (a) $K(u, 2.5)$ and its corresponding fish boundary; (b) $K(u, 20)$ and its corresponding fish boundary; (c) $K(u, 40)$ and its corresponding fish boundary.
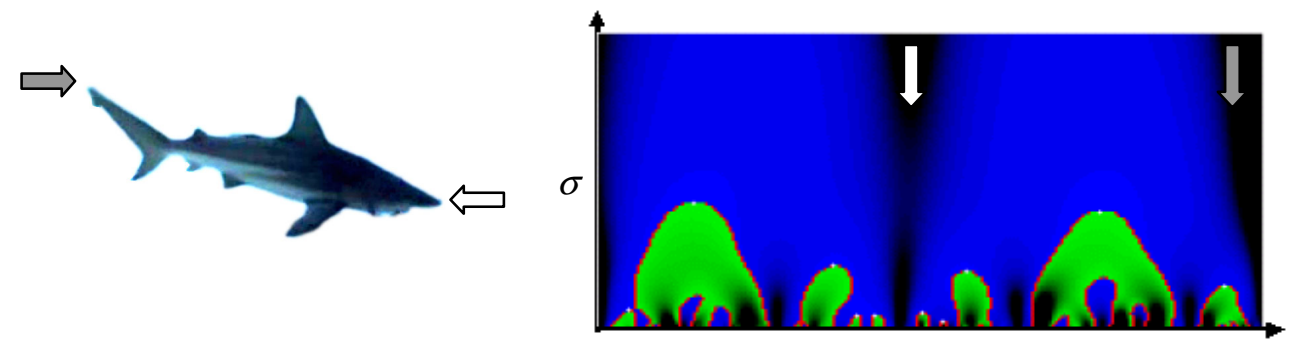

(a)

$u$ (arc length)

Fig. 5. Relative position of the fish head and tail in the CSS image. 

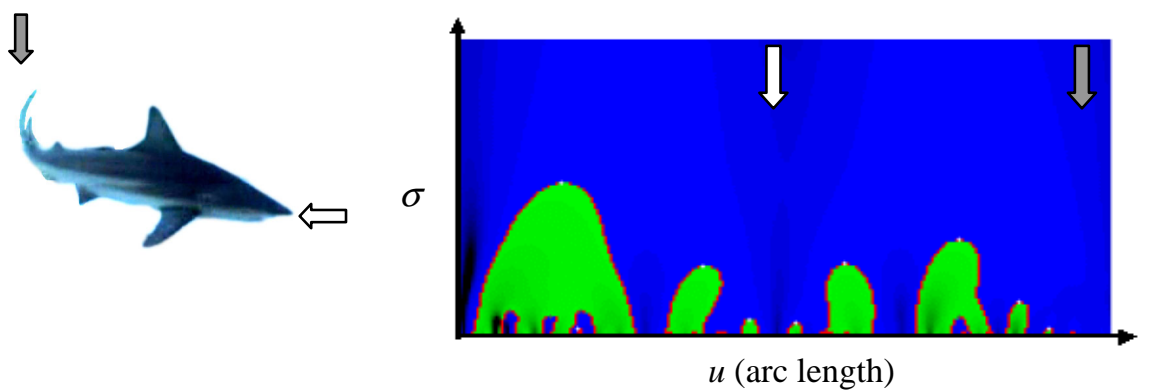

(b)

Fig. 5. (Cont'd) Relative position of the fish head and tail in the CSS image.

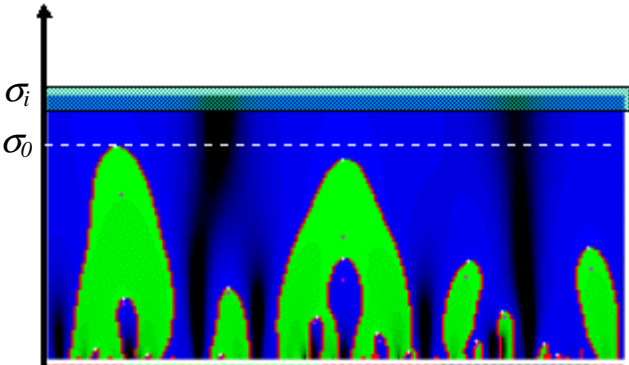

$u$ (arc length)

(a)

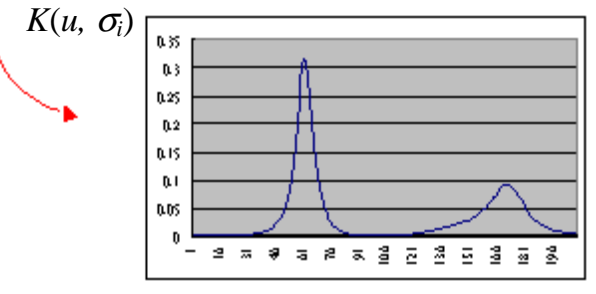

(b)

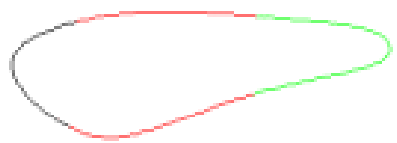

(c)

Fig. 6. (a) A CSS image; (b) the curvature function at $\sigma_{i}$; (c) the fish boundary at $\sigma_{i}$.

\subsection{CSS-Based Motion Model}

In this section, we shall describe a CSS-based motion model for a fish to be built. We shall use the rectangular object shown in Fig. 8(a) to explain the model. For the rectangular object shown in Fig. 8(a), 232 boundary points are needed to describe its shape. After Gaussian convolution is performed at $\sigma=35$, the shape boundary of the object degenerates into an ellipse, as shown in Fig. 8(b). The horizontal and vertical lines indicate the long and short axes of the ellipse, respectively. Moreover, the curvature function contains two local maxima $(u=68,182)$ and two local minima $(u=10,125)$, as shown in Fig. 8(c). The two local maxima indicate the long axis of the ellipse, while the two local minima indicate the short axis. Before the object rotates around its vertical axis, its corresponding projection area is maximal. When the object rotates 90 degrees, its corresponding projection area becomes minimal. When the object rotates 180 or 360 degrees, 


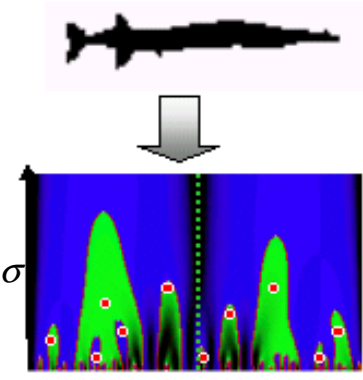

$u$ (arc length)
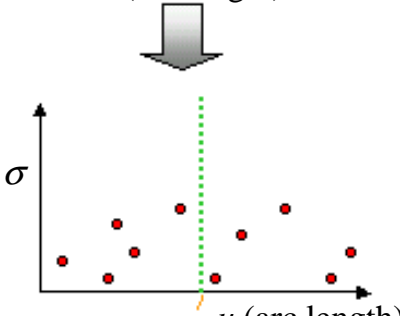

$u$ (arc length)

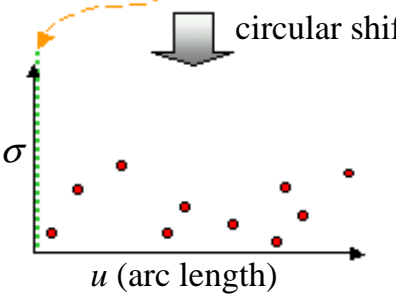

Fig. 7. Matching two fishes after circular shifting of maximum points.

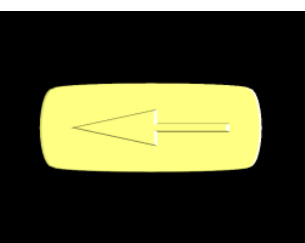

(a)

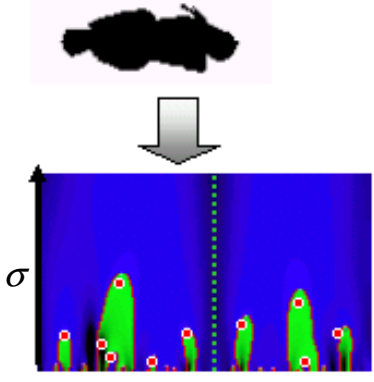

$u$ (arc length)
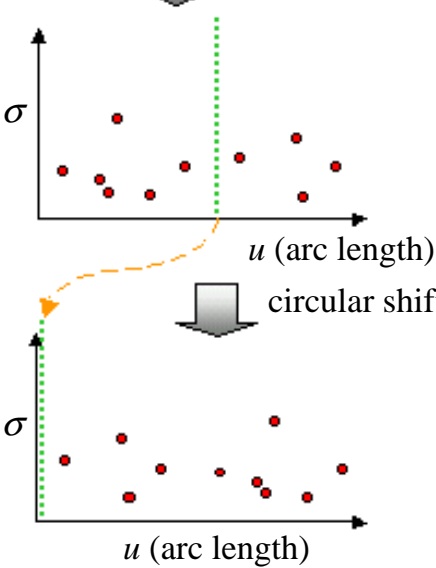

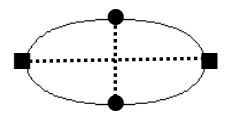

(b)

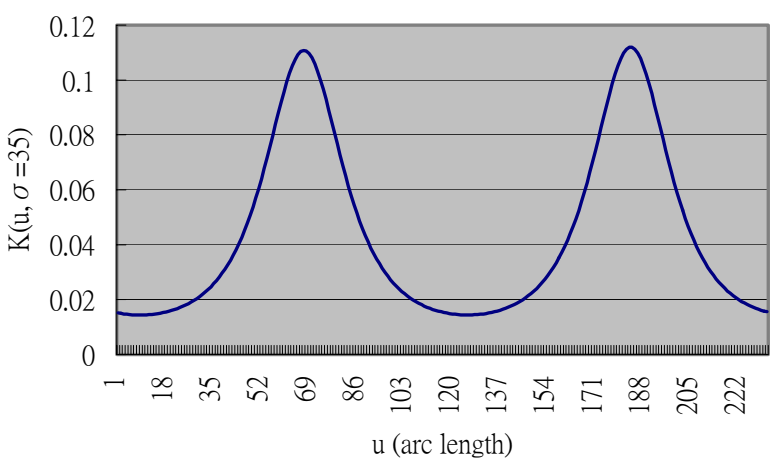

(c)

Fig. 8. (a) A rectangle-like object with 232 boundary points; (b) the ellipse and its long and short axes; (c) the corresponding curvature function at $\sigma=35$. 
its corresponding projection area reaches the maximum again. Fig. 9 shows a number of projections obtained from different angles of rotation.

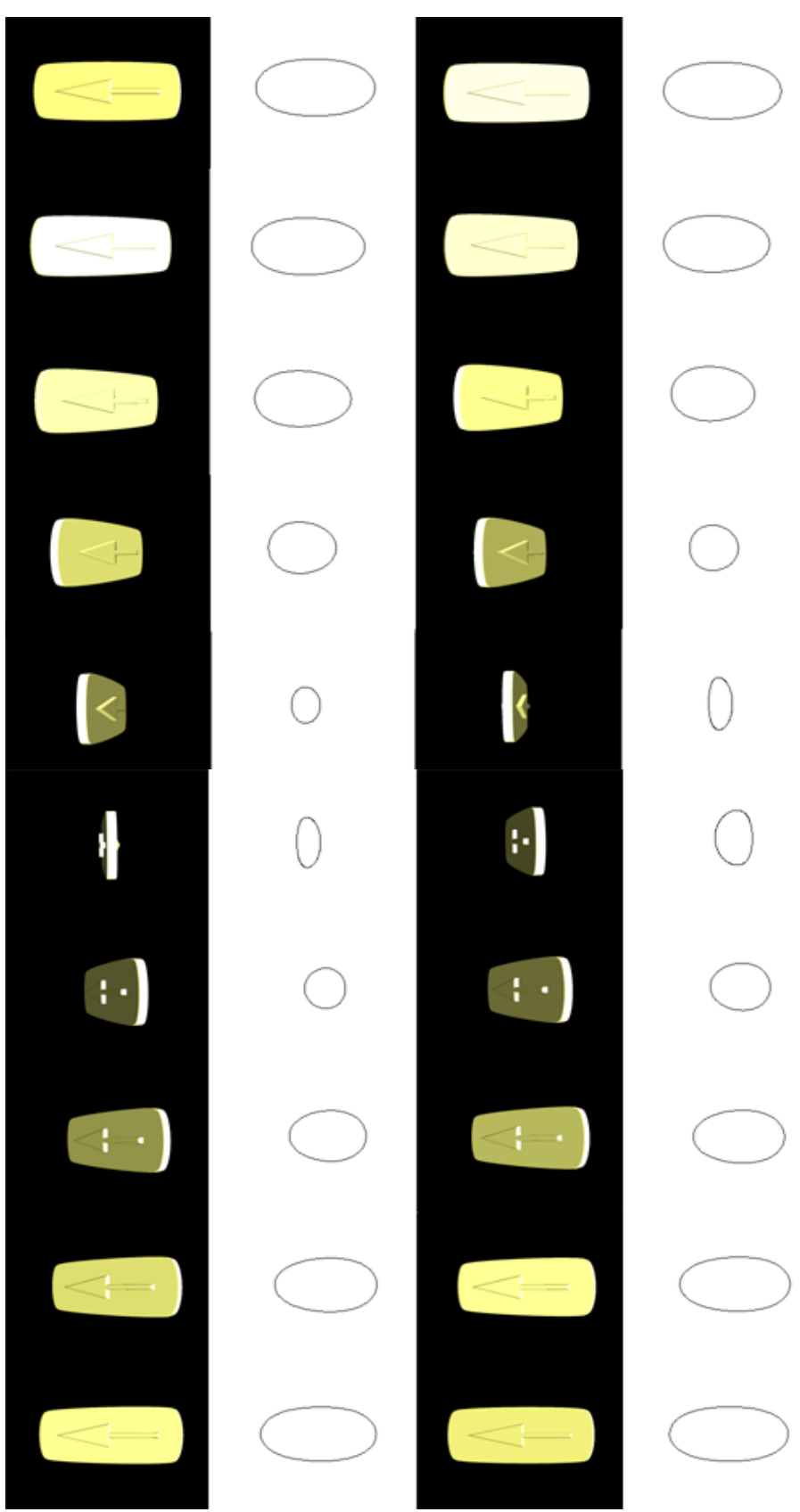

Fig. 9. Rotating a rectangle-like object (from left to right and top to bottom) and its corresponding degenerate boundary at $\sigma=35$. 

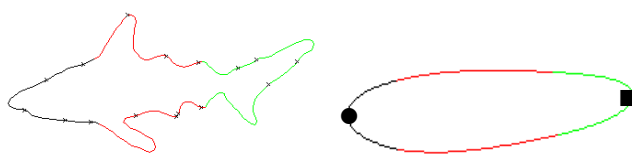

(a)
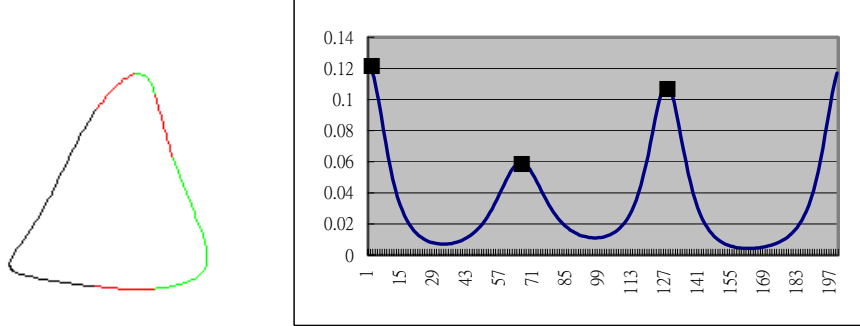

(b)

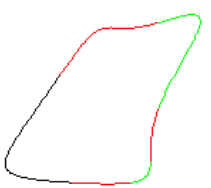

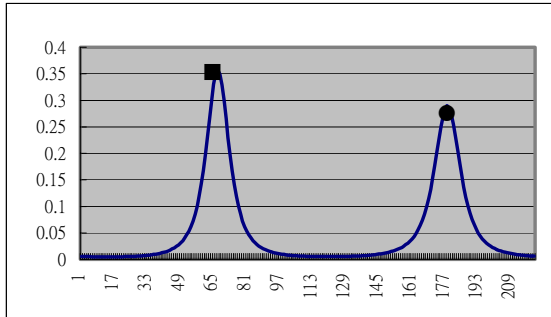

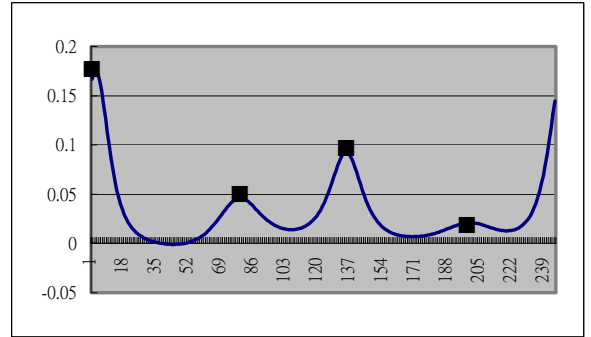

(c)

Fig. 10. (a) Quasi-ellipse at $\sigma_{i}=1.25 \sigma_{0}$ and $P=2$; (b) the degenerated boundary obtained at $\sigma_{i}$ and $P=3$; (c) the degenerated boundary obtained at $\sigma_{i}$ and $P=4$.

Recall that we only collect fish whose length is at least twice its width. Therefore, the bounding parallelogram of a fish can always be assumed to be a rectangle. After Gaussian convolution is conducted at a specific value of $\sigma$, the fish boundary may degenerate into a quasi-ellipse. Suppose we are observing a moving fish, which may swing its tail fin to move forward or turn around. When the fish is recorded in an image sequence, its poses in the images are all different. For example, Fig. 10 shows three different fish poses. When swimming toward to our left, the fish is in left view, as shown in Fig. 10(a). The fish boundary degenerates to a quasi-ellipse at $\sigma_{i}$, and the curvature function contains only two local maxima. However, when turning around, the fish is in front (or rear) view, as shown in Figs. 10(b) and 10(c). The fish boundary may suffer serious deformation or be occluded by other parts of the fish. Therefore, the fish boundary does not degenerate into a quasi-ellipse at $\sigma_{i}$, and the curvature function contains more than two motion models for a moving fish, as shown in Fig. 11. The fish 
two motion models for a moving fish, as shown in Fig. 11. The fish poses are classified into three types, namely left, right, and front (or rear) views. To formalize this, let $P$ be the number of local maxima in the curvature function after Gaussian convolution is performed at $\sigma_{i}$. The value of $P$ can be used to determine a fish pose as follows: If $P=2$, the fish is in left or right view, whereas if $P>2$, the fish is in front (or rear) view. It is clear that the fish with side view is easier to recognize than that in front (or rear) view.
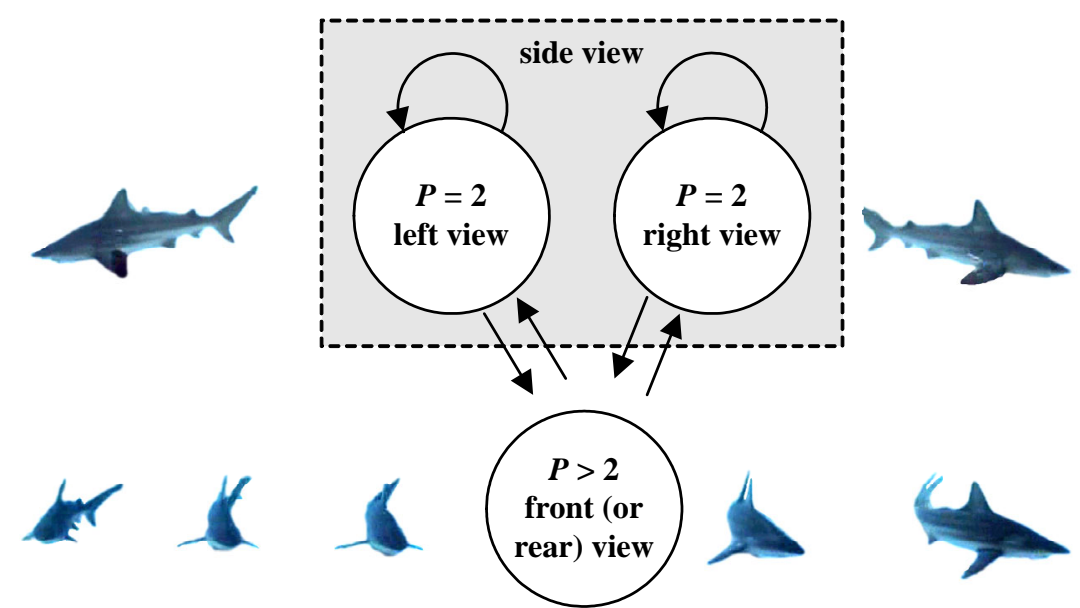

Fig. 11. Motion model for a moving fish.

\subsection{CSS-Based Matching}

Assume that there is a sequence of $k$ images of a moving fish that are in left view. After the CSS image creation process is performed, we can obtain $k$ sets of maxima points. Let $S_{j}$ be the $j$-th set of maxima points, where $1 \leq j \leq k$, and let $n_{j}$ be the number of maxima points in $S_{j}$. We have

$$
S_{j}=\left\{\left(u_{l}^{j}, \sigma_{l}^{j}\right) \mid 1 \leq l \leq n_{j}\right\},
$$

where $\left(u_{l}^{j}, \sigma_{l}^{j}\right)$ denotes the $l$-th maxima point in $S_{j}$. The average maxima points for the sequence of $k$ images can be derived by executing the c-means algorithm as follows:

\section{Algorithm 1. C-means algorithm.}

Input. $k$ sets of maxima points.

Output. A set of average class centers, $Z_{\text {ave }}$.

Step 1. Let the $m$-th set have the largest number of maxima points in the $k$ sets of maxima points, and let $c$ be the number of classes and $Z$ be the set of class centers. Initialize $c=n_{m}$ (where $n_{m}$ is the number of elements in the $m$-th set) and $Z=\left\{\left(u_{l}^{m}, \sigma_{l}^{m}\right) \mid 1 \leq l \leq n_{m}\right\}$. 
Step 2. For each set of maxima points, classify each point into one of the $c$ classes according to the minimum distance criterion.

Step 3. For each class, a new center can be obtained by averaging the maxima points that are classified into the class (in Step 2). Let $Z_{\text {ave }}$ be the set of average class centers. If $Z_{\text {ave }} \neq Z$ then set $Z=Z_{\text {ave }}$ and go to Step 2 .

\section{Stop.}

In this study, the average class centers will be used as the representative maxima points for the sequence of $k$ images during the matching process. Note that if a sequence of images of a fish are with right view, their maxima points should be horizontally flipped in advance because we assume that the default view is left view.

The process of matching an unknown image sequence (i.e., the query) with each database image is executed by comparing the average class centers with maxima points of the fish. Let $S$ be the set of maxima points for a fish in the database. Each maxima point in $S$ is classified into one of the $c$ classes based on the minimum distance criterion. The matching score is defined as the sum of the Euclidean distance from each maxima point to its nearest class center. The smaller the matching score is, the more similar the fish described by the image sequence is to the fish in the database.

\section{EXPERIMENTAL RESULTS}

In the experiments, we used a fish image database in Taiwan that contains 2000 fish species as the underlying database. Note that we only collected fish whose length was at least twice large its width. In addition, only left views (headed toward the left) were used as database images. The test image sequence was obtained from Discovery Channel Video KDV-4063 [28], which introduces the ecological environment of tiger sharks. The number of frames (images) in the sequence was 30, each of which was sampled from the video every 0.2 seconds. Fig. 12 shows the 30 test images, which were numbered 0-29 from left to right and top to bottom. Fig. 13 shows the results after executing our foreground/background separation algorithm [21].

Based on the poses extracted from the tiger shark, the test images can be classified into three types, including "move leftward" (Nos. 0-3), "turn around" (Nos. 4-12), and "move rightward" (Nos. 13-29). Fig. 14 shows the classification results for the 30 test images. According to the value of $P$, the test sequence could be divided into three subsequences, namely, Nos. 0-3, Nos. 4-12, and Nos. 13-29. If $P=2$, the tiger shark was in left or right view; therefore, the images of Nos. 0-3 and Nos. 13-29 were reserved for matching. If $P>2$, the tiger shark was in front (or rear) view; therefore, the images of Nos. 4-12 were discarded. According to the relative positions of two curvature maxima, we could determine the direction toward which the tiger shark was moving.

We now had two image subsequences with the side view, namely, Nos. 0-3 (left view) and Nos. 13-29 (right view). For each image in a sequence, we generated its corresponding CSS image and then extracted maxima points from it. The maxima points were then regularized through circular shift and height adjustment. For all the images in a 


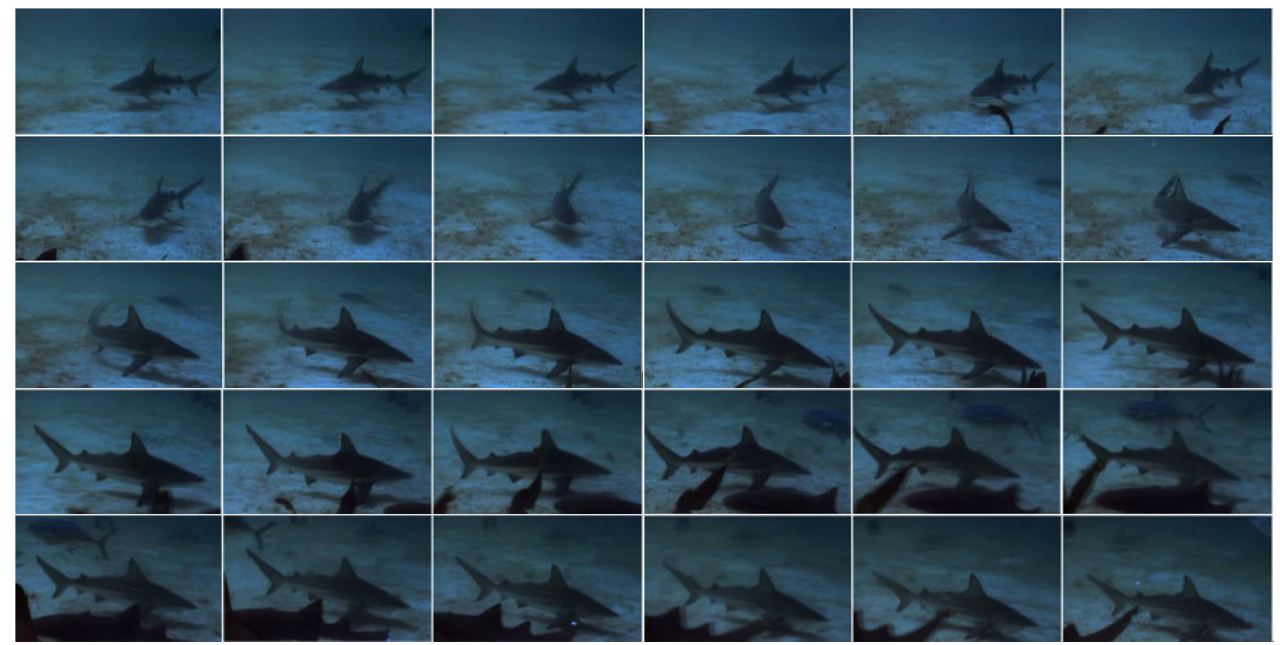

Fig. 12. A sequence of 30 test images of a moving tiger shark.

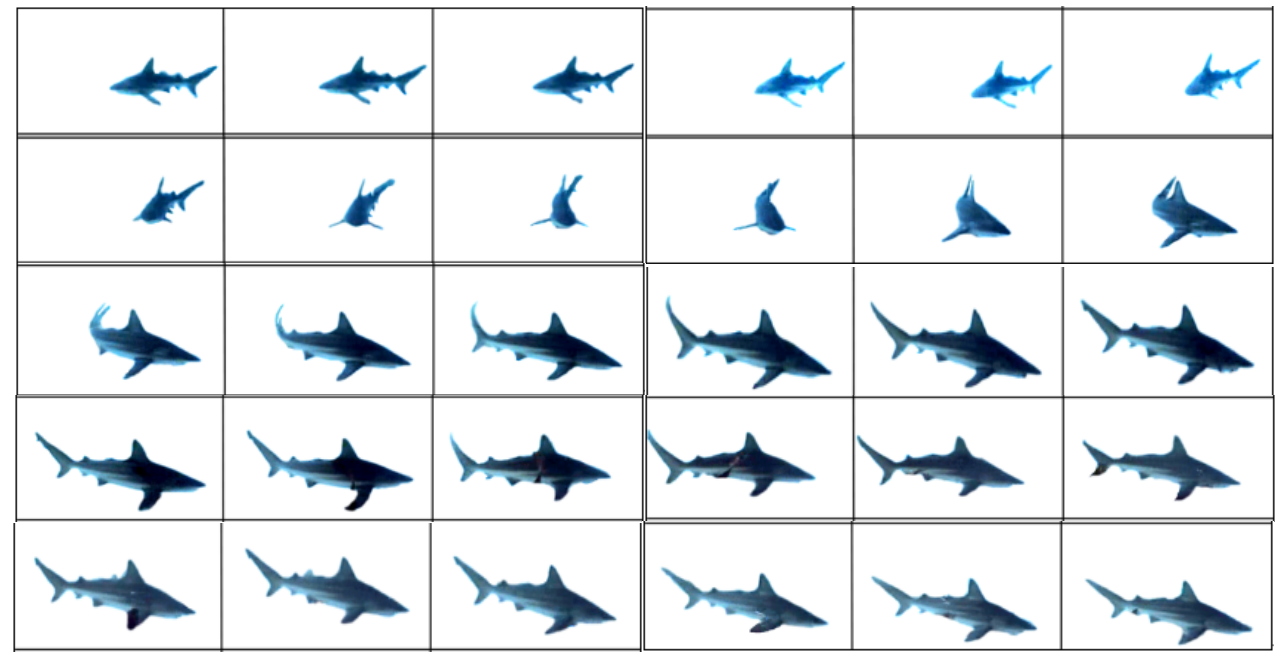

Fig. 13. The 30 test images after performing foreground/background separation.

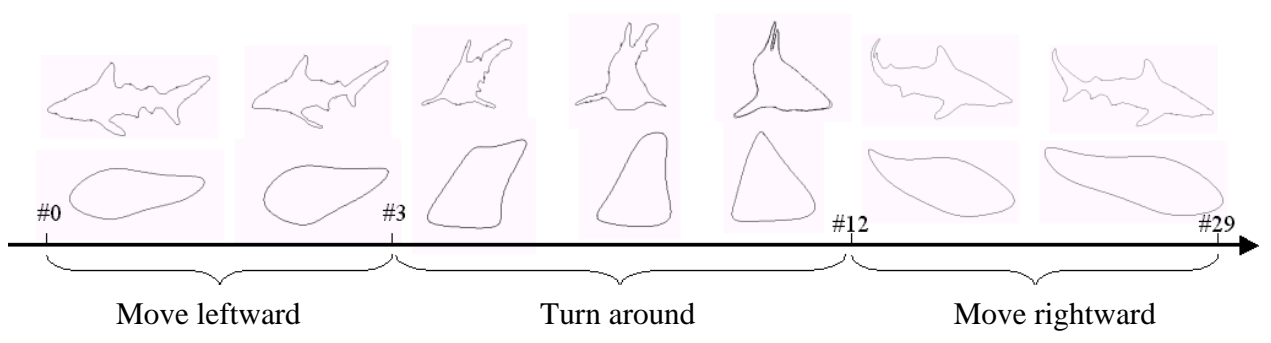

Fig. 14. Classification results for the 30 test images. 


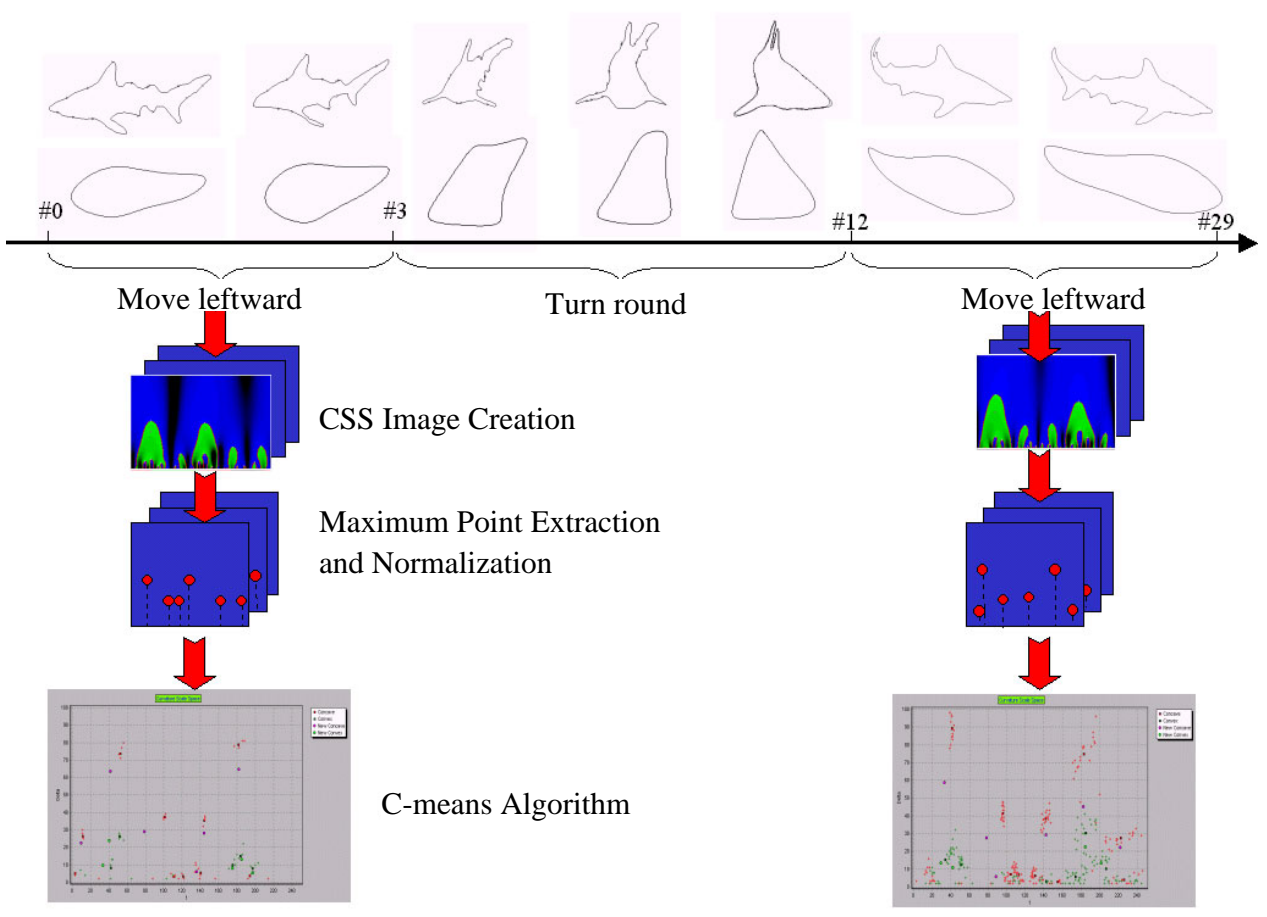

Fig. 15. An illustration showing how side views can be converted into a representative feature vector using the c-means algorithm.

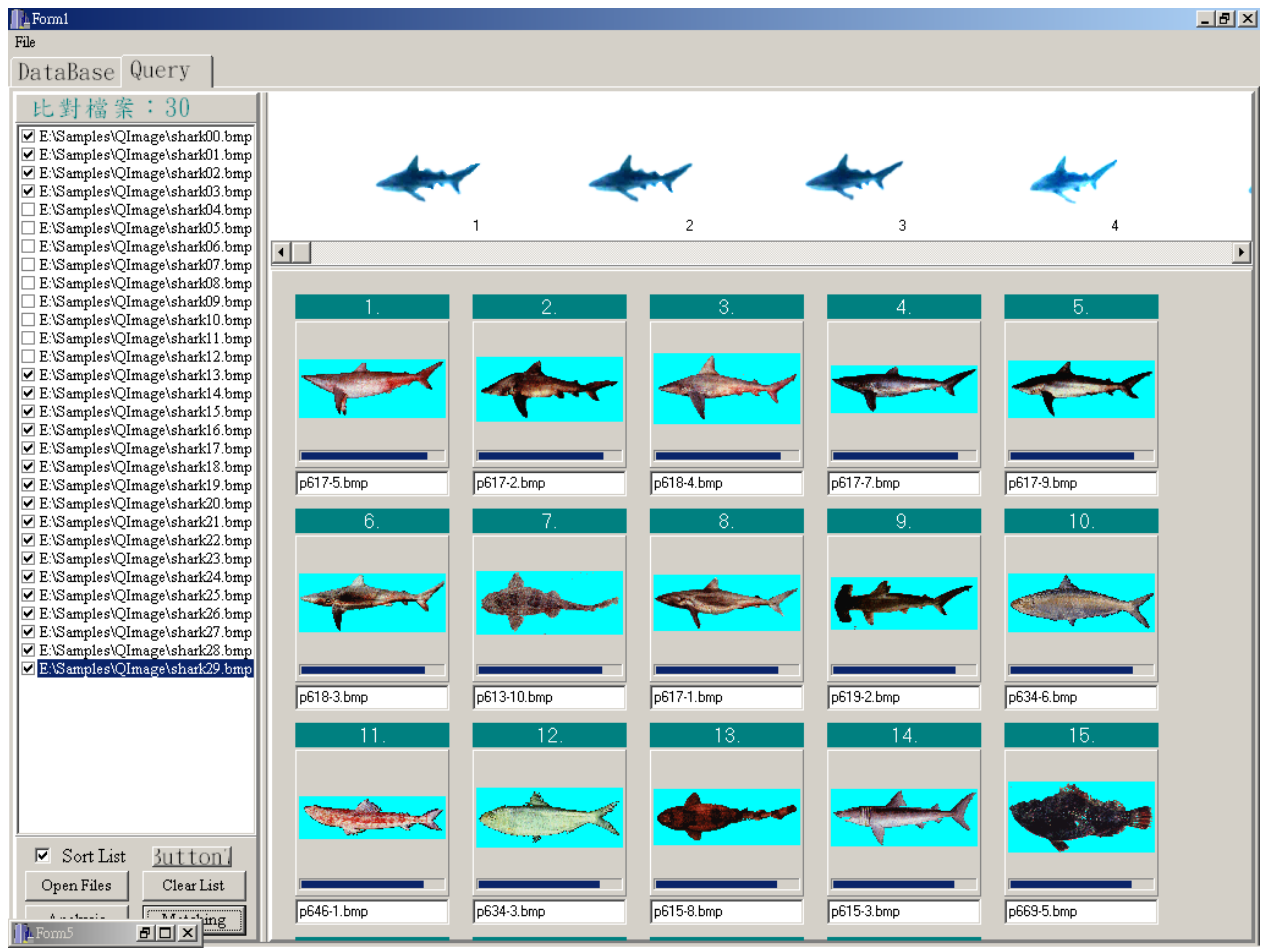

Fig. 16. The retrieval results. 
sequence, we computed their average maxima points using the c-means algorithm. Fig. 15 shows how side views were converted into a representative feature vector using the c-means algorithm. Fig. 16 shows the retrieval results for the provided sequence. The retrieved fish images are displayed in descending order based on the degree of similarity from left to right and top to bottom. Users can click each retrieved fish image for getting further textual descriptions. According to the above experimental results, it is obvious that our approach is indeed a superb one.

\section{CONCLUSIONS}

A content-based image retrieval (CBIR) system based on features extracted from a video clip has been proposed in this paper. The proposed system has been tested using a fish database collected in Taiwan. The contribution of this work is three-fold. First, our query by video content scheme can overcome problems caused by non-rigid fish motion, serious deformations, and partial occlusion. Second, our query scheme can overcome problems caused by varying imaging conditions. Third, our CSS-based representation scheme is invariant to translation, scaling, and rotation. Experimental results strongly supported the above-mentioned claims.

\section{REFERENCES}

1. A. D. Bimbo, Visual Information Retrieval, Morgan Kaufmann Publishers, 1999.

2. P. Aigrain, H. J. Zhang, and D. Petkovic, "Content-based representation and retrieval of visual media: a state-of-the-art review," Multimedia Tools and Applications, Vol. 3, 1996, pp. 179-202.

3. S. F. Chang, J. R. Smith, H. J. Meng, H. Wang, and D. Zhong, "Finding images/video in large archives: Columbia's content-based visual query project," Digital Library Magazine, Vol. 3, 1997.

4. A. Gupta and R. Jain, "Visual information retrieval," Communication of the ACM, Vol. 40, 1997, pp. 70-79.

5. F. Idris and S. Panchanathan, "Review of image and video indexing techniques," Journal of Visual Communication and Image Representation, Vol. 8, 1997, pp. 146-166.

6. Y. Rui, T. S. Huang, and S. F. Chang, "Image retrieval: current techniques, promising directions, and open issues," Journal of Visual Communication and Image Representation, Vol. 10, 1999, pp. 39-62.

7. A. W. M. Smeulders, M. Worring, S. Santini, A. Gupta, and R. Jain, "Content-based image retrieval at the end of the early years," IEEE Transactions on Pattern Analysis and Machine Intelligence, Vol. 22, 2000, pp. 1349-1380.

8. A. A. Goodrum, "Image information retrieval: an overview of current research," Informing Science, Vol. 3, 2000, pp. 63-67.

9. L. G. Shapiro and G. C. Stockman, Computer Vision, Prentice Hall, 2001.

10. M. Bober, "MPEG-7 visual shape descriptors," IEEE Transactions on Circuits and Systems for Video Technology, Vol. 11, 2001, pp. 716-719.

11. A. Pentland, R. Picard, and S. Sclaroff, "Photobook: tools for content-based manipu- 
lation of image databases," Storage and Retrieval for Image and Video Database II, SPIE Proceedings, Vol. 2185, 1994, pp. 34-47.

12. S. Sclaroff, A. Pentland, and R. W. Picard, "Photobook: content-based manipulation of image databases," International Journal of Computer Vision, Vol. 18, 1996, pp. 233-254.

13. C. Faloutsos, W. Equitz, M. Flickner, W. Niblack, D. Petkovic, and R. Barber, "Efficient and effective querying by image content," Research Report, No. 9453, IBM Almaden Research Center, 1989.

14. R. Barber, W. Equitz, C. Faloutsos, M. Flickner, W. Niblack, D. Petkovic, and P. Yanker, "Query by content for large on-line image collections," Research Report, No. 9408, IBM Almaden Research Center, 1993.

15. W. Niblack and M. Flickner, "Find me the pictures that look like this: IBM's image query project," Advanced Imaging, Vol. 8, 1993, pp. 32-35.

16. W. Niblack, R. Barber, W. Equitz, M. Flickner, E. Glasman, and D. Petkovic, "The QBIC project: querying images by content using color, texture, and shape," Research Report, No. 9203, IBM Almaden Research Center, 1993.

17. M. Flickner, H. Sawhney, W. Niblack, J. Ashley, Q. Huang, B. Dom, M. Gorkani, J. Hafner, D. Lee, D. Petkovic, D. Steele, and P. Yanker, "Query by image and video content: the QBIC system," IEEE Computers, Vol. 28, 1995, pp. 23-32.

18. D. H. Huang and C. L. Huang, "A content-based image retrieval system," IPPR Conference on Computer Vision, Graphics, and Image Processing, 1996, pp. 259-266.

19. W. I. Grosky and Y. Lu, "Iconic indexing using generalized pattern matching techniques," Computer Vision, Graphics, and Image Processing, Vol. 35, 1996, pp. 383-403.

20. A. D. Bimbo and P. Pala, "Visual image retrieval by elastic matching of uers sketches," IEEE Transactions on Pattern Analysis and Machine Intelligence, Vol. 19, 1997, pp. 121-132.

21. C. J. Sze, H. R. Tyan, H. Y. Liao, C. S. Lu, and S. K. Huang, "Shape-based retrieval on a fish database of Taiwan," Tamkang Journal of Science and Engineering, Vol. 2, 1999, pp. 163-173.

22. S. Abbasi, F. Mokhtarian, and J. Kittler, "Curvature scale space image in shape similarity retrieval," Springer Journal of Multimedia Systems, Vol. 7, 1999, pp. 467-476.

23. H. C. Lin, L. L. Wang, and S. N. Yang, "Fast heuristics for polygonal approximation of a 2D shape boundary," Signal Processing, Vol. 60, 1997, pp. 235-241.

24. A. P. Witkin, "Scale-space filtering," International Joint Conference on Artificial Intelligence, 1983, pp. 1019-1022.

25. A. P. Witkin, "Scale-space filtering: a new approach to multi-scale description," Image Understanding, 1984, pp. 79-95.

26. N. Ansari and E. J. Delp, "On detecting dominant points," Pattern Recognition, Vol. 24, 1991, pp. 441-450.

27. S. Abbasi, F. Mokhtarian, and J. Kittler, "Scale similarity retrieval using a height adjusted curvature scale space image," International Conference on Visual Information Systems, 1997, pp. 173-180.

28. Discovery Channel Video, "Secret of the deep II - sand tigers," KDV-4036, 1999. 


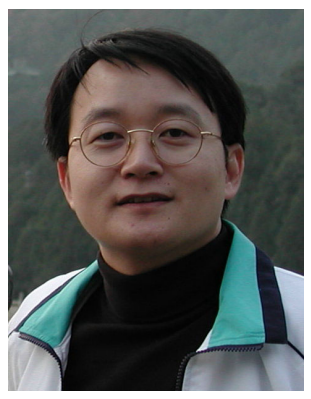

Hsin-Chih Lin (林信志) was born in Tainan County, Taiwan in 1968. He received the B.E. degree in Electrical Engineering from National Tsing Hua University, Hsinchu, Taiwan in June 1990 and the Ph.D. degree in Computer Science from the National Tsing Hua University in June 1997. Since August 1999, he joined the faculty of the Department of Information Management, Chang Jung Christian University (CJCU), Tainan County, Taiwan as an Assistant Professor.

Dr. Lin was a recipient of the Research Award of National Science Council in 1999, and the Annual Research Award of CJCU from 2001 to 2002. Dr. Lin was also a recipient of the Distinguished Teaching Award and the Distinguished Counseling Award at CJCU in 2002. His research interests include computer graphics, image understanding, multimedia information retrieval, and web mining.

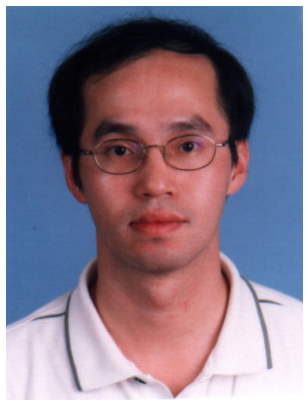

Arthur Chun-Chieh Shih (施純傑), also known as ChwenJye Sze, was born Taipei, Taiwan, on September 26, 1966. He received the B.S. degree in electrical engineering from the Chinese Culture University, Taipei, Taiwan, in 1992, the M.S. degree also in electrical engineering from National Chung-Cheng University, Chiayi, Taiwan, in 1994 and a Ph.D. degree in computer science and information engineering from National Central University, Chung-Li, Taiwan, in 1998. Form October 1998 to July 2002, he worked for Institute of Information Science, Academia Sinica, Taiwan and the Department of Ecology and Evolution, the University of Chicago, USA as postdoctoral fellows, one after another. He joined Institute of Information Science, Academia Sinica, Taiwan, as an assistant research fellow in July 2002. His current research interests include multimedia processing and bioinformatics.

Hsiao-Rong Tyan (田篌榮) received the B.S. degree in electronic engineering from Chung-Yuan Christian University, Chung-Li, Taiwan, in 1984, and the M.S. and Ph.D. degrees in computer science from Northwestern University, Evanston, IL, in 1987 and 1992, respectively. She is an Associate Professor of the Department of Information and Computer Engineering, Chung-Yuan Christian University, Chungli, Taiwan, where she currently conducts research in the areas of computer networks, computer security, and intelligent systems. 


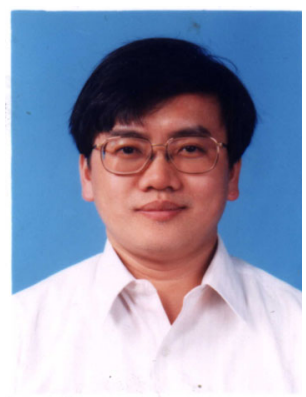

Chi-Fang Lin (林啓芳) was born in 1960 in Taiwan, Republic of China. He received the B.S. degree in Transportation Engineering and Management in 1983, and the M.S. and the Ph. D. degrees in Institute of Computer Engineering in 1986 and 1991, all from National Chiao Tung University.

During his study at National Chiao Tung University, he worked in the school library as a system engineer from 1983 to 1986. In the academic year 1987-1989, he was an instructor in Lien Hu Junior College of Technology. He was a chairman of Department of Information Networking Technology at Yuan-Ze University, and also was the director of Information Technology Research Center of the same University during the years from 1999 to 2002. He is currently a professor in the Department of Computer Engineering and Science at Yuan-Ze University. His field interest includes image processing, pattern recognition, and computer vision.

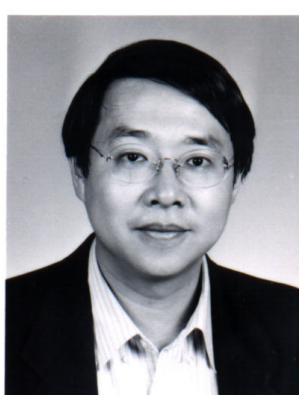

Mark Liao (廖弘源) received the B.S. degree in physics from National Tsing-Hua University, Hsinchu, Taiwan, in 1981, and the M.S. and Ph.D. degrees in electrical engineering from Northwestern University, Illinois, in 1985 and 1990, respectively.

He was a research associate in the Computer Vision and Image Processing Laboratory at Northwestern University during 1990-1991. In July 1991, he joined Institute of Information Science, Academia Sinica, Taiwan, as an assistant research fellow. He was promoted to associate research fellow and then research fellow in 1995 and 1998, respectively. From August 1997 to July 2000, he served as the deputy director of the institute. Currently, he is the acting director of Institute of Applied Science and Engineering Research, Academia Sinica, Taiwan.

Dr. Liao's current research interests include multimedia signal processing, wavelet-based image analysis, content-based multimedia retrieval, and multimedia protection. He was the recipient of the Young Investigators' award of Academia Sinica in 1998; the excellent paper award of the Image Processing and Pattern Recognition society of Taiwan in 1998 and 2000; Dr. Liao served as the program chair of the International Symposium on Multimedia Information Processing (ISMIP'1997) and the program co-chair of the second IEEE Pacific-Rim Conference on Multimedia (2001). He will serve as a conference co-chair of the IEEE International Conference on Multimedia and Exposition at 2004. He also served on the program committees of several international and local conferences. Dr. Liao was on the Editorial Board of the IEEE Transactions on Multimedia (1998-2001). He is now on the Editorial Boards of the International Journal of Visual Communication and Image Representation; the Acta Automatica Sinica, and the Tamkang Journal of Science and Engineering. He is now the managing editor of the Journal of Information Science and Engineering. He is a senior member of the IEEE. 